\title{
Why do bubbles in Guinness sink?
}

\author{
E. S. Benilov C. P. Cummins
}

\begin{abstract}
Stout beers show the counter-intuitive phenomena of sinking bubbles while the beer is settling. Previous research suggests that this phenomena is due the small size of the bubbles in these beers and the presence of a circulatory current, directed downwards near the side of the wall and upwards in the interior of the glass. The mechanism by which such a circulation is established and the conditions under which it will occur has not been clarified. In this paper, we demonstrate using simulations and experiment that the flow in a glass of stout depends on the shape of the glass. If it narrows downwards (as the traditional stout glass, the pint, does), the flow is directed downwards near the wall and upwards in the interior and sinking bubbles will be observed. If the container widens downwards, the flow is opposite to that described above and only rising bubbles will be seen.
\end{abstract}

\section{INTRODUCTION}

Stout beers, such as Guinness, foam due to a combination of dissolved nitrogen and carbon dioxide, $\frac{1}{-}$ as opposed to other beers which foam due to dissolved carbon dioxide alone. The use of nitrogen results in a range of desirable characteristics of the beer, including a less bitter taste and a creamy long-lasting head which can be attributed to the low solubility of nitrogen and small size of the bubbles. ${ }^{2,3}$ This small bubble size is also responsible, at least in part, for another intriguing characteristic of stout beers: the phenomenon of sinking bubbles, observed while the beer is settling, i.e. between the pouring of the beer and the formation of the head. $\underline{4}$

Experimental studies ${ }^{5}$ have demonstrated that the phenomenon of sinking bubbles is real and not an optical illusion, while simulations $\frac{6}{-}$ show that the bubbles are driven by a downward flow, the velocity of which exceeds the upward velocity of the bubble due to the Archimedean force. The existence of such a flow near the wall of the glass implies that there must be an upward flow somewhere in the interior. The mechanism of this circulation is, however, unclear, as is the role of the shape of the glass.

Understanding these types of bubbly flows is important for a number of applications, such as manufacturing champagne glasses engraved with nucleation sites, $\underline{7}$ widget and similar technologies for promoting foaming in stouts,,$\frac{8,9}{9}$ designing glasses which minimize the settling time of stouts and, generally, for industrial processes involving bubbly flows (e.g. bubble columns ${ }^{10}$ ).

In this paper, we put forward an explanation of the effect of sinking bubbles in Guinness, which takes into account the role of the shape of the glass. First, in Section II] we describe the properties of Guinness as a twophase medium. In Section III we present the results of numerical simulations for several shapes of the glass. In Section IV we explain the basic mechanism which drives bubbles downwards and describe a simple experiment which can be used to confirm our hypothesis. Finally we give conclusions in Section $\mathrm{V}$.

\section{PROPERTIES OF GUINNESS}

We shall model Guinness by a liquid of density $\rho_{l}$ and viscosity $\mu_{l}$, with randomly distributed bubbles of gas of density $\rho_{g}$ and viscosity $\mu_{g}$. For a temperature of $6^{\circ} \mathrm{C}$ (recommended for consumption of Guinness by its producer "Diageo"12) and normal atmospheric pressure, we have

$$
\begin{aligned}
\rho_{l} & =1007 \mathrm{~kg} \mathrm{~m}^{-3} & \mu_{l} & =2.06 \times 10^{-3} \mathrm{Pas} \\
\rho_{g} & =1.223 \mathrm{~kg} \mathrm{~m}^{-3} & \mu_{g} & =0.017 \times 10^{-3} \mathrm{Pas}
\end{aligned}
$$

where the former values have been measured by ourselves and verified against the extrapolation formula of Ref. 4 .

To check whether the bubble shapes differ from spheres, we introduce the Bond number

$$
\mathrm{Bo}=\frac{\rho_{l} g d_{b}^{2}}{\sigma}
$$

where $d_{b}$ is the bubbles' characteristic diameter, $\sigma$ is the surface tension of the liquid/gas interface, and $g$ is the acceleration due to gravity. Assuming $d_{b}=122 \mu \mathrm{m}$ (as reported in Ref. 11) and $\sigma=0.745 \mathrm{Nm}^{-1}$ (which corresponds to water/air interface), we obtain Bo $\approx 0.002$ - which is sufficiently small to assume that bubbles in Guinness are spherical.

Note also that Guinness (as well as vast majority of 'real' liquids) contains a lot of surfactants, which make the bubbles behave as rigid spheres. 13 This allows one to estimate the characteristic bubble velocity $u_{b}$ using the Stokes formula for a rigid sphere,

$$
u_{b}=\frac{\left(\rho_{l}-\rho_{b}\right) g d_{b}^{2}}{18 \mu_{l}} \approx 3.96 \mathrm{mms}^{-1}
$$

Estimating the corresponding Reynolds number

$$
\mathrm{Re}=\frac{\rho_{l} u_{b} d_{b}}{\mu_{l}} \approx 0.24
$$

confirms that the Stokes formula yields a qualitatively correct value for $u_{b}$. Furthermore, given that $u_{b}$ is much smaller than the speed of sound, the gas can be treated as incompressible. 
Finally, we introduce the void fraction $f$, i.e. the gas's share of the volume of the liquid/gas mixture. For canned Guinness, $f \approx 0.05$ (see Ref. 11), whereas for draught Guinness served in pubs, $f \approx 0.1$ (according to our own measurements). Note, however, that, traditionally, bartenders first fill, say, $80 \%$ of the glass and wait until it has fully settled (i.e. all the bubbles have gone out of the liquid into the foamy head), after which they would fill the glass full. Thus, when Guinness is served to the customer, the void fraction can be estimated as $f \approx 0.02$, which is the value used in this work.

\section{NUMERICAL MODELING OF THE LIQUID/BUBBLE CIRCULATION}

To simulate flows in Guinness, we use the finite element model for bubbly flows included in the COMSOL Multiphysics package. The model's physical foundations are described in detail in Ref. 16. In this model the bubbles are assumed to be all of the same size. In view of the problem's axial symmetry, the axi-symmetric version of the model is used.

Two geometries of the holding container were examined (see Fig. 1): a pint and an 'anti-pint', i.e. the pint turned upside-down. In both cases the initial distribution of bubbles was uniform, and the physical parameters of Guinness were as described above.

The results of typical simulations are shown in Fig. 1 One can see that an elongated vortex arises near the sloping part of the pint container, resulting in a downflow of bubbles along the wall (see the top-left panel of Fig. 2). A similar vortex also exists in the anti-pint, but it rotates in the opposite direction and, thus, causes an upward flow near the wall (see the top-right panel of Fig. 2).

Another important feature to be observed is the narrow region of low density of bubbles along the wall of the pint container (see the left panel of Fig. 1] and the lowerleft panel of Fig. 2). In the anti-pint container, in turn, the bubble density increases near the wall (which is not visible in the right panel of Fig. 1, but can be clearly seen in the lower-right panel of Fig. 2).

We have also examined the evolution of the global void fraction for the pint and anti-pint, as well as the cylindrical container of the same volume. Fig. 3 shows that all three geometries provide more or less the same settling time $T_{s}$ (for a glass of stout, a smaller $T_{s}$ is generally regarded as an advantage).

$T_{s}$ has also been used to examine the extent to which our results depend on the void fraction $f$ and the bubble size $d_{b}$ (these are the only parameters with 'uncertain' values). It has turned out that, surprisingly, the dependence of $T_{s}$ on $f$ is very weak: e.g., an increase in $f$ from 0.02 to 0.05 results in a decrease in $T_{s}$ from 43 to 46 seconds (the settling time here was defined such that $\left.f\left(T_{s}\right)=10^{-6}\right)$. The dependence of $T_{s}$ on $d_{b}$ is much stronger: a decrease in $d_{b}$ from 122 to 90 micron results in an increase in $T_{s}$ from 43 to 83 seconds.
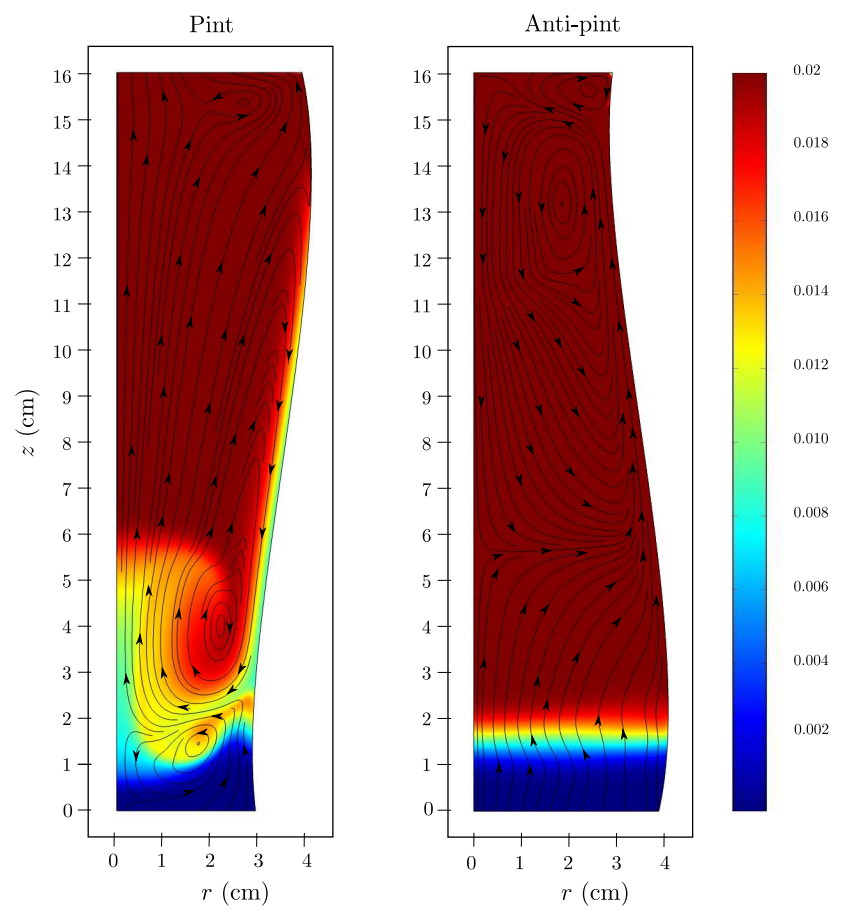

FIG. 1. Numerical simulations of bubbly flows for the pint and anti-pint. The curves show the streamlines for the bubbles, the color shows the void fraction $f$. The snapshots displayed correspond to $t=4 \mathrm{~s}$. Observe the region of reduced $f$ near the wall of the pint (the near-wall region of increased $f$ in the anti-pint is not visible in this figure, but can be observed in Fig. 2].

Note also that the settling time of, approximately, 43 seconds computed for $d_{b}=122 \mu \mathrm{m}$ and the pint container does not agree with our experimental estimate of approximately 120 seconds. The difference between the two results is probably caused by the fact that all bubbles in our simulations were of the same size, whereas in reality they are distributed with a certain dispersion. One can then conjecture that smaller bubbles leave the liquid later than those of the median size, which would account for the difference between the computed and measured values of $T_{s}$.

In what follows, we shall argue that the circulation developing in the flow is determined by the near-wall variation of the bubble density (as suggested previously by Ref. 14 for bubble columns), and that the bubble density, in turn, is determined by the shape of the container.

\section{THE MECHANISM OF THE EFFECT}

First of all, observe that, whichever way the bubbles move, they exert a drag force on the surrounding liquid. This does not mean, however, that the liquid is necessarily entrained by the motion of the bubbles. Indeed, if, for example, the bubbles (and, hence, the drag force) are distributed uniformly, all liquid particles must move 


\section{Pint}
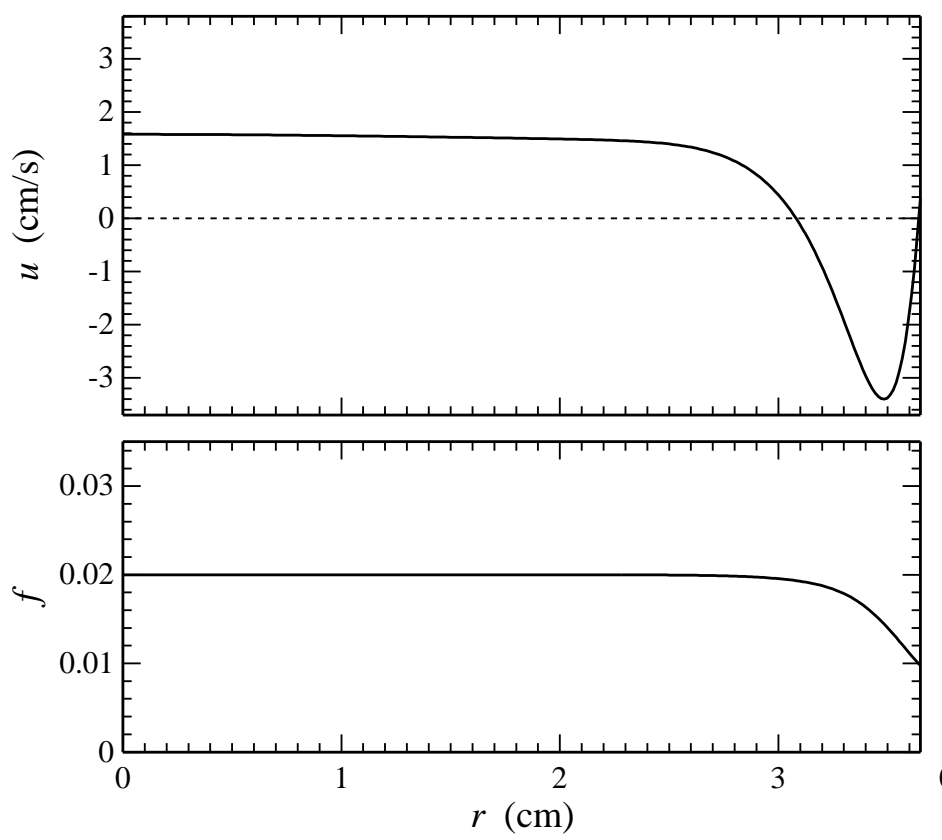

Anti-pint
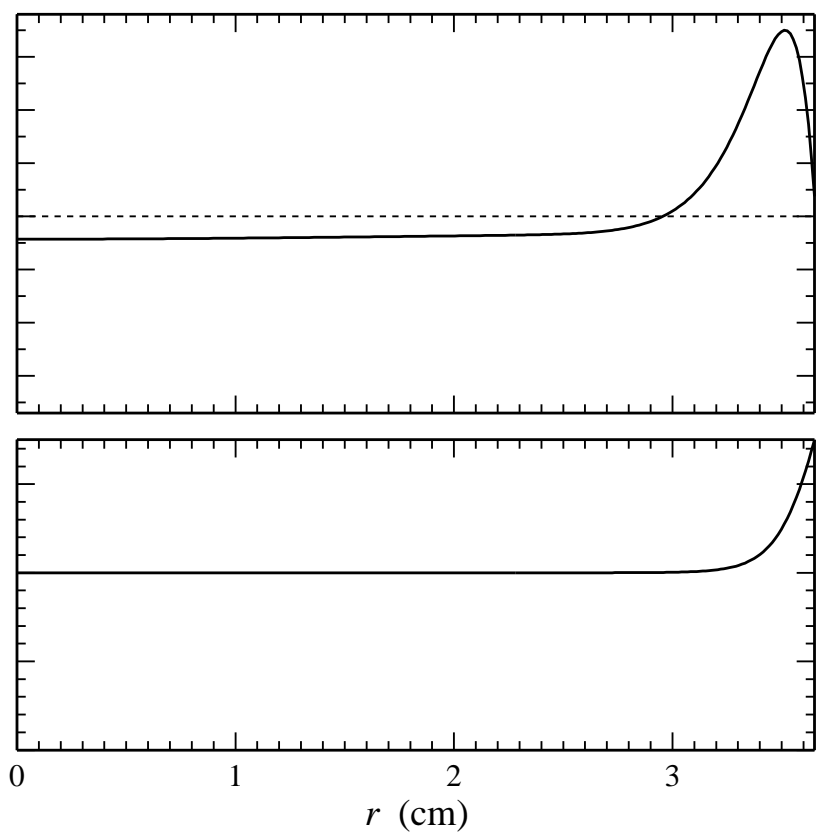

FIG. 2. The half-height cross-sections of the vertical velocity $u$ and the void fraction $f$ for the pint and anti-pint geometries (these graphs correspond to the $(r, z)$ diagrams shown in Fig. 2). The dotted lines in the upper panels separate the regions of upward/downward flow.

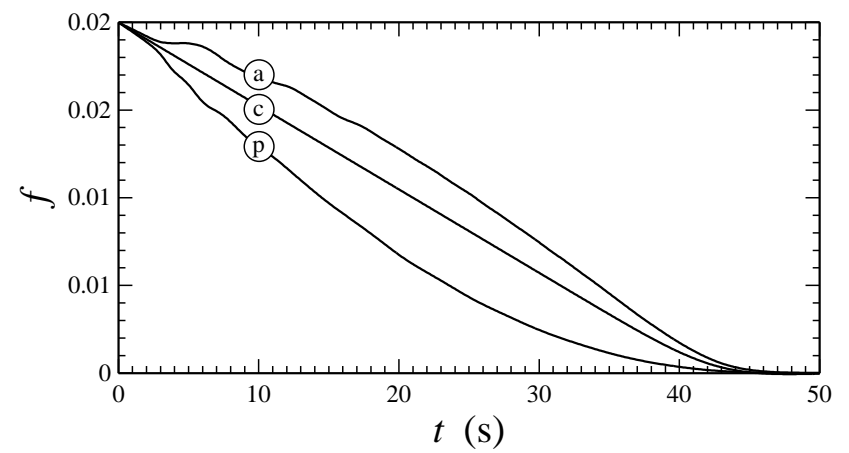

FIG. 3. The global void fraction $f$ (i.e. the proportion of the gas in the container) versus time $t$, for the three cases: the pint (p), anti-pint (a) - both illustrated in Fig. 2-and a cylinder of the same volume (c).

the same way - which effectively means that they cannot move at all due to the liquid's incompressibility and the fact that the container has a bottom. In this case, the drag force is compensated by a pressure gradient exerted in the fluid.

Let us now assume that there is a region of low bubble density near the container's wall (as there indeed is in the pint container). In this case, the density of the drag force near the container's axis is larger than that near the wall - which creates an imbalance and, thus, gives rise to a circulation: near the axis, the liquid flows upwards and, near the wall, downwards. Then, if the velocity of the downward flow is larger than the relative velocity $u_{b}$ of the bubbles, the bubbles will be observed to sink. A similar argument indicates that a near-wall region with higher bubble density gives rise to an upward flow (i.e. exactly what our simulations show for the anti-pint container).

It still remains to identify the mechanism reducing the bubble density near the wall for the pint geometry and increasing it for the anti-pint one. Potentially, there are two such mechanisms. The most obvious one is based on the 'lift force', generated by the flow around a sphere moving along a rigid boundary: this force pushes the sphere away from the boundary $\underline{15}$. In the limit of small Reynolds number, however, the lift force is weak, and estimates show that the resulting reduction of the bubble density near the wall is negligible. Another shortcoming of this mechanism is that it does not seem to distinguish between the pint and anti-pint geometries.

To explore the effect of the geometry, assume that the container is not cylindrical, but narrows slightly towards its bottom (as the pint does). Then, even if the bubbles were initially distributed uniformly, their upward motion immediately creates a bubble-free zone along the wall (see Fig. 4). On the other hand, in a container that widens towards its bottom (as the anti-pint does), the initially upward motion of bubbles increases the nearwall bubble density. We believe that this simple kinematic effect is responsible for the circulation observed in Guinness.

This effect, although not previously discussed in the 


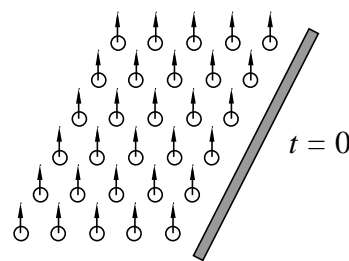

(a)

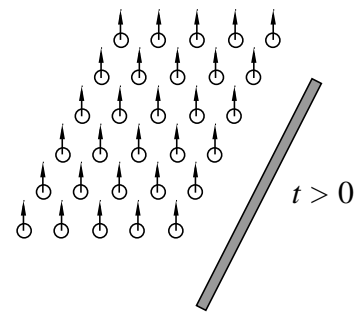

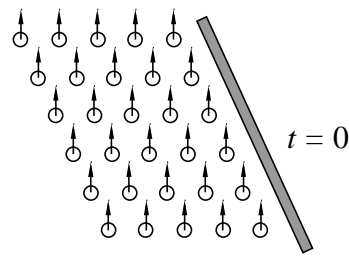

(b)

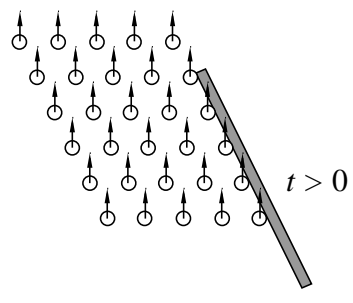

FIG. 4. The evolution of bubbles near the wall (a schematic), for (a) container narrowing downwards (the bubbles move away from the wall); (b) container widening downwards (the bubbles move towards the wall and, eventually, accumulate there).

context of Guinness, is well known in sedimentation theory as the Boycott effect. $\frac{17,18}{1 t}$ was first observed in test tubes containing red blood cells when it was discovered that sedimentation times could be significantly reduced by inclining the test tubes.
Finally, our conclusions can be readily verified experimentally. If Guinness is poured into a tall cylindrical container (such as, for example, a laboratory measuring cylinder) and if the container is tilted, bubbles will be observed to move upwards near its upper surface and downwards near its lower surface - in precise agreement with the mechanism proposed (see supplementary material for a video of this experiment).

\section{CONCLUSIONS}

The sinking bubbles of Guinness and other stout beers have intrigued beer drinking physicists and their students for some time. In this paper we describe the role the shape of the Guinness pint glass plays in promoting the circulatory flow responsible for the sinking bubbles. In doing so we complete the explanation of this phenomena, building on previous experimental and simulation work. This allows us to understand the physics underlying the shape of the Guinness pint glass. It also raises the intriguing question: is this shape the most efficient possible or could the settling time be significantly reduced by some other, possibly non-axisymmetric, shape of pint glass?

\section{ACKNOWLEDGMENTS}

The authors acknowledge the support of the Science Foundation Ireland delivered through RFP Grant 11/RFP.1/MTH3281 and Mathematics Initiative Grant 06/MI/005.
* Department of Mathematics, University of Limerick, Ireland; $\quad$ Eugene.Benilov@ul.ie http://www.staff.ul.ie/eugenebenilov/hpage/

$\dagger$ Department of Mathematics, University of Limerick, Ireland;Cathal.Cummins@ul.ie

¥ MACSI, Department of Mathematics, University of Limerick, Ireland; William.Lee@ul.ie; http://www.ul.ie/wlee/

1 W. T. Lee and M. G. Devereux, "Foaming in stout beers," Am. J. Phys. 79, 991 (2011).

2 M. Denny, Froth!: the science of beer (Johns Hopkins University Press, Baltimore, 2009).

${ }^{3}$ C. W. Bamforth, "The Relative Significance of Physics and Chemistry for Beer Foam Excellence: Theory and Practice," J. Inst. Brew. 110, 259-266 (2004).

$4 \mathrm{Y}$. Zhang and Z. Xu, ""Fizzics" of beer and champagne bubble growth," Elements 4, 47 (2008).

5 A. C. Alexander and R. N. Zare, "Do bubbles in Guinness go down?" http://www.stanford.edu/group/Zarelab/guinness/

6 R. F. Service, "The Unbuoyant Bubbles of Guinness," ScienceNOW, http://news.sciencemag.org/sciencenow/2000/01/06-02.html (2000).

7 G. Liger-Belair, J. B. Religieux, S. Fohanno, M. A. Vialatte, P. Jeandet, and G. Polidori, "Visualization of mixing flow phenomena in champagne glasses under various glass-shape and engravement conditions," J. Agric. Food Chem., 55, 882-888 (2007).

8 W. T. Lee, J. S. McKechnie, and M. G. Devereux, "Bubble nucleation in stout beers," Phys. Rev. E 83, 051609 (2011).

9 E. Gerstner, "Bubble nucleation: Stout fizz-ics," Nature Physics 7, 449 (2011).

10 A. Lapin, T. Paaschen, K. Junghans, and A. Lubbert, "Bubble column fluid dynamics," Chem. Eng. Sci. 57, 1419 (2002).

11 M. Robinson, A. C. Fowler, A. J. Alexander, and S. B. G. O'Brien, "Waves in Guinness," Phys. Fluids 20, 067101 (2008).

12 FAQ, Guinness Storehouse, http://www.guinness-storehouse.com/en/FAQs . aspx\#faq23

13 G. K. Batchelor, An introduction to fluid dynamics (Cambridge University Press, Cambridge 2000).

14 N. Rivière, A. Cartellier, L. Timkin, and O. Kashinsky, "Wall shear stress and void fraction in Poiseuille bubbly flows: Part II: experiments and validity of analytical predictions," Eur. J. Mech. B 18, 847 (1999).

1]F. Takemura and J. Magnaudet, "Lateral migration of a small spherical buoyant particle in a wall-bounded linear shear flow," Phys. Fluids 21, 1 (2009). 
16 A. Sokolichin, G. Eigenberger, and A. Lapin, "Simulation of buoyancy driven bubbly flow: Established simplifications and open questions," AIChE J. 50, 24 (2004).
17 A.E.Boycott, "Sedimentation of blood corpuscles" Nature 104, 532 (1920).

18 A. Acrivos and E. Herbolzheimer, "Enhanced sedimentation in settling tanks with inclined walls" Journal of Fluid Mechanics 92, 435 (1979). 